\title{
Quantum Information Processing Using the Exchange Interaction
}

\author{
M. Guha Majumdar \\ Cavendish Laboratory, University of Cambridge, Cambridge, UK \\ Email:mg712@cam.ac.uk
}

How to cite this paper: Majumdar, M.G. (2018) Quantum Information Processing Using the Exchange Interaction. Journal of Quantum Information Science, 8, 139-160. https://doi.org/10.4236/jqis.2018.84010

Received: September 9, 2018

Accepted: November 19, 2018

Published: November 22, 2018

Copyright (C) 2018 by author and Scientific Research Publishing Inc. This work is licensed under the Creative Commons Attribution International License (CC BY 4.0).

http://creativecommons.org/licenses/by/4.0/

\begin{abstract}
In this paper, I propose new models of quantum information processing using the exchange interaction in physical systems. The partial SWAP operator that can be realized using the exchange interaction is used as the underlying resource for defining models of quantum computation, quantum communication, quantum memory and decoherence-free subspaces. Given the noncommutativity of these operators (for adjacent operators operating on a common qubit), a number of quantum states and entanglement patters can be obtained. This zoo of states can be classified, due to the parity constraints and permutation symmetry of the states, into invariant subspaces that are used for the definition of some of the applications in this paper.
\end{abstract}

\section{Keywords}

Entanglement, Quantum Computation, Quantum Communication

\section{Introduction}

Entanglement is a non-classical manifestation of quantum formalism that was formulated to describe the non-local correlations that develop in quantum systems in certain physical processes and systems [1]-[7]. Over the years, this facet of the quantum world has been found in a number of physical systems, be it photonic [8] [9] [10] [11], phononic [12] [13] [14], atomic [15] [16] [17] [18] [19] or electronic [20] [21] [22]. Entanglement is also the quintessential quantum-mechanical phenomenon that forms the underlying resource for future quantum technologies and therefore is a major subject of interest and research [23]-[28]. Be it in quantum computation [29] [30] [31], quantum communication [32] [33] [34] or quantum cryptography [35] [36] [37], entanglement is what these applications in quantum information processing are built on.

Entanglement in condensed matter systems, from quantum dots [38] [39] and 
semiconductor heterostructures [40] to Nitrogen-vacancy centers [41] [42], have been used to develop quantum technologies using entangled many-body systems [4] [5] [6]. Among the various physical interactions that can generate entanglement, the exchange interaction

$$
H=J \vec{S}_{1} \cdot \vec{S}_{2}
$$

has been particularly useful for generating entanglement [38]. Here $J$ is the coupling constant and $\vec{S}$ are the spins in the interaction. The exchange operation can generate the $\sqrt{S W A P}$ as well as the $S W A P^{\alpha}$ gates [43] [44]. Burkard et al. [45] showed how an XOR (Exclusive OR) gate can be created from a CPHASE (Controlled Phase-Flip) gate that can be created using the $\sqrt{\text { SWAP }}$ gate. Subsequently, Barenco et al. [46] showed that two XOR gates and four one-bit gates can simulate any unitary two-qubit gate, thereby paving the way for realizing universal quantum computation. Divincenzo et al. [26] showed that universal quantum computation can be achieved using only the exchange interaction if one considers logical qubits to be the fundamental building blocks of the computational system. Another way to realize universal quantum computation that can be implemented using the exchange interaction is the cluster state quantum computation model. Tanamoto et al. [47] showed that upon preparing the initial state $| \pm\rangle=\frac{1}{\sqrt{2}}(0 \pm 1)$ and applying the $\sqrt{S W A P}$ one can obtain the familiar Raussendorf's cluster state [48] after the operation of two single-qubit rotations. Given the general non-commutativity of $S W A P^{\alpha}$ gates (for adjacent operators operating on a common qubit), the number of states that can be generated with the operation of these operators are many times more than what could be achieved using a commutative operator such as the Controlled-Phase Gate. However, due to the parity constraints and permutation symmetries of states operated upon by these operators, one can classify the states into invariant subspaces, which can be used for various tasks in quantum information processing.

In this paper, I look at various quantum information processing applications that have been devised independently. In Section 2, quantum computation using the $S W A P^{\alpha}$ gate is discussed using five different models. In Section 3, we look at entanglement swapping, repeaters and quantum communication using the exchange interaction. In Section 4, we propose a way to implement quantum memory using the $S W A P^{\alpha}$ gate arising out of the exchange interaction. In Section 5, we look at the idea of decoherence-free subspaces and how this can be defined for systems undergoing the exchange interaction between its constituents.

\section{Quantum Computing Using SWAP ${ }^{\alpha}$}

Quantum Computation is the manipulation of quantum resources and quantum entanglement therein for the purposes of realizing an information processing task. Historically, the circuit-based model of quantum computation and mea- 
surement-based model of quantum computation have been the most popular. These arise from the key concepts of evolution and measurement of a quantum particle or system. The $S W A P^{\alpha}$ gate is a powerful tool for carrying out quantum computation, due to its ubiquity in physical systems such as those with exchange interactions. We have seen that the locus of states accessible using these gates is restricted to a certain subspace of the Hilbert space. As a result, it is understood that not all states are accessible by only using the $S W A P^{\alpha}$ gate.

In this section, we will be looking at how to realize five different models of quantum computation using the $S W A P^{\alpha}$ gate as the key cornerstone of this discussion.

\subsection{Circuit-Based Quantum Computing}

In the realm of quantum information, a quantum circuit model of quantum computation is one wherein a computation is a sequence of quantum gates. These quantum gates are reversible transformations on a quantum register, a system comprising multiple qubits. The key paradigm shift, going from classical computation to quantum computation is the presence of reversible (quantum) logic gates. These mappings preserve the Hermitian inner product and a general $n$-qubit (reversible) quantum gate is a unitary mapping $U$ from the Hilbert space of n-qubits onto itself. The pertinent point to be addressed here is regarding the number of quantum gates and resources required that can optimally approximate any quantum computation.

\section{Universal Gate Set}

A set of universal quantum gates is a set of quantum gates that can, in a finite sequence of gates from this set, replicate any arbitrary unitary operation that may be possible on a quantum computer [49] [50] [51] [52]. For physical systems with exchange interaction, universal quantum gates have been constructed with encoded qubits [26] [53], while the Loss-Divincenzo Quantum Computer relies on the $\sqrt{S W A P}$ and single-qubit gates [38]. DiVincenzo and Loss showed that the $\sqrt{S W A P}$ gate is universal with single-qubit rotations. This universality is derived in terms of the relation of the $\sqrt{S W A P}$ gate with the classical XOR gate, which can be realized using the CNOT gate in the realm of quantum information processing [54]. This leads us to believe that a generalized case (of any general $S W A P^{\alpha}$ can comprise a universal gate set too).

The first step in defining a universal gate set using $S W A P^{\alpha}$ is to realize that no such set can be made purely out of $S W A P^{\alpha}$ gates since these gates preserve Hamming weight of the quantum state representation. If we allow single qubit unitary operations, let us see the lowest number of $S W A P^{\alpha}$ that are required to carry this out. Given this symmetry of the $S W A P^{\alpha}$ gates, the cases we look into are: $A_{1}|00\rangle+B_{1}|11\rangle \rightarrow A_{2}|00\rangle+B_{2}|11\rangle,\left|A_{1}\right|^{2}+\left|B_{1}\right|^{2}=\left|A_{2}\right|^{2}+\left|B_{2}\right|^{2}=1$ and $C_{1}|01\rangle+D_{1}|10\rangle \rightarrow C_{2}|01\rangle+D_{2}|10\rangle, \quad\left|C_{1}\right|^{2}+\left|D_{1}\right|^{2}=\left|C_{2}\right|^{2}+\left|D_{2}\right|^{2}=1$. However, how is this possible if the $S W A P^{\alpha}$ gate leaves the states $|00\rangle$ and $|11\rangle$ unchanged? This can be done by using a qubit-flip gate on one of the qubits: 


$$
\begin{aligned}
U_{1} & =U_{S W A P^{\alpha_{1}}}\left(\sigma_{x} \otimes I_{2 \times 2}\right) U_{S W A P^{\alpha_{2}}} \\
& =\left(\begin{array}{cccc}
0 & \frac{1-\mathrm{e}^{i \pi \alpha_{2}}}{2} & \frac{1+\mathrm{e}^{i \pi \alpha_{2}}}{2} & 0 \\
\frac{1-\mathrm{e}^{i \pi \alpha_{1}}}{2} & 0 & 0 & \frac{1+\mathrm{e}^{i \pi \alpha_{1}}}{2} \\
\frac{1+\mathrm{e}^{i \pi \alpha_{1}}}{2} & 0 & 0 & \frac{1-\mathrm{e}^{i \pi \alpha_{1}}}{2} \\
0 & \frac{1+\mathrm{e}^{i \pi \alpha_{2}}}{2} & \frac{1-\mathrm{e}^{i \pi \alpha_{2}}}{2} & 0
\end{array}\right)
\end{aligned}
$$

The $S W A P^{\alpha}$ also has a fixed accessibility of states, as mentioned previously in this paper. Since the sum of the coefficients for vectors with the same Hamming weight add to the same value over an operation of $S W A P^{\alpha}$ gates, states that do not follow this rule cannot be accessed. To begin with, two Bell-states differing by a relative phase of $\mathrm{e}^{i \pi}$ cannot be inter-converted using $S W A P^{\alpha}$ gates. This can, however, be achieved using a phase-flip operator on a single qubit, say the second qubit.

$$
\begin{aligned}
U_{2} & =U_{1}\left(I_{2 \times 2} \otimes \sigma_{z}\right) \\
& =\left(\begin{array}{cccc}
0 & \frac{-1+\mathrm{e}^{i \pi \alpha_{2}}}{2} & \frac{1+\mathrm{e}^{i \pi \alpha_{2}}}{2} & 0 \\
\frac{1-\mathrm{e}^{i \pi \alpha_{1}}}{2} & 0 & 0 & \frac{-1-\mathrm{e}^{i \pi \alpha_{1}}}{2} \\
\frac{1+\mathrm{e}^{i \pi \alpha_{1}}}{2} & 0 & 0 & \frac{-1+\mathrm{e}^{i \pi \alpha_{1}}}{2} \\
0 & \frac{-1-\mathrm{e}^{i \pi \alpha_{2}}}{2} & \frac{1-\mathrm{e}^{i \pi \alpha_{2}}}{2} & 0
\end{array}\right)
\end{aligned}
$$

Even though this brings in the extreme case of $\left|\psi_{ \pm}\right\rangle \rightarrow\left|\psi_{m p}\right\rangle$ and $\left|\phi_{ \pm}\right\rangle \rightarrow\left|\phi_{m p}\right\rangle$, there are lots of other states that should be accessible using a general two qubit unitary gate. This greater independence is seen to come from the application of yet another $S W A P^{\alpha}$ gate:

$$
\begin{aligned}
U_{3} & =U_{2} U_{S W A P^{\alpha_{3}}} \\
& =\left(\begin{array}{cccc}
0 & \frac{\mathrm{e}^{i \pi \alpha_{2}}-\mathrm{e}^{i \pi \alpha_{3}}}{2} & \frac{\mathrm{e}^{i \pi \alpha_{2}}+\mathrm{e}^{i \pi \alpha_{3}}}{2} & 0 \\
\frac{1-\mathrm{e}^{i \pi \alpha_{1}}}{2} & 0 & 0 & \frac{-1-\mathrm{e}^{i \pi \alpha_{1}}}{2} \\
\frac{1+\mathrm{e}^{i \pi \alpha_{1}}}{2} & 0 & 0 & \frac{-1+\mathrm{e}^{i \pi \alpha_{1}}}{2} \\
0 & \frac{-\mathrm{e}^{i \pi \alpha_{2}}-\mathrm{e}^{i \pi \alpha_{3}}}{2} & \frac{-\mathrm{e}^{i \pi \alpha_{2}}+\mathrm{e}^{i \pi \alpha_{3}}}{2} & 0
\end{array}\right)
\end{aligned}
$$

This along with local unitary operations $\left\{K_{i}, L_{i}\right\}$, where the index $i$ denotes the qubit being operated on, should be able to implement any general two-qubit quantum gate. Hence, in the most general form, any two qubit quantum gate can be realized by the expansion 


$$
\left(K_{1} \otimes K_{2}\right)\left(U_{S W A P^{\alpha_{1}}}\left(\sigma_{x} \otimes I_{2 \times 2}\right) U_{S W A P^{\alpha_{2}}}\left(I_{2 \times 2} \otimes \sigma_{z}\right) \times U_{S W A P^{\alpha_{3}}}\right)\left(L_{1} \otimes L_{2}\right)
$$

Any circuit with two-qubit and single-qubit gates can thus be constructed using the $S W A P^{\alpha}$ gate, alongwith single-qubit unitary operations.

\subsection{Invariant Subspace-Based Quantum Computing}

Permutation symmetry has been of significant interest to the world of quantum physics and quantum information [55] [56] [57] [58] [59]. An early example of the success of the use of permutation-symmetric states was in the discovery of noiseless subspaces [56], in which quantum states could evolve without the introduction of any bit-flip errors. This follows from the constraints on the parity of the states that belong to these subspaces. Considering binary quantum states, a (partial) permutation on two qubits can be carried out by a (partial) SWAP gate [60], which as we saw previously can be realized using the exchange interaction. Due to this parity constraint for permutation-symmetric quantum states, one can define certain invariant subspaces that are constituted by vectors that remain invariant under the operation of permutation operators. For instance, the completely symmetric n-qubit Dicke states (e.g. $\frac{1}{\sqrt{3}}(|001\rangle+|010\rangle+|100\rangle)$ for $n=3$ and Hamming weight 1 in the state representation) will remain invariant under any permutation operator, and hence constitute a one-dimensional invariant subspace. Similarly one can compose higher dimensional invariant subspaces that are composed of vectors whose linear combination remains invariant under specific permutation operators.

We see that this manner of composing invariant subspaces using the permutation symmetry of the system can be used for defining a model of quantum computation based on these subspaces. For defining the model, let us look at the fundamental elements that are needed to create such an implementation. The three most important sections of a quantum computer are: high-fidelity initialization of the input quantum state, detection by measurement of the output quantum state at the individual qubit level and control of operations by interactions between qubits. In the previous section, we have defined universality of a set of quantum gates comprising the $S W A P^{\alpha}$ gate and quantum single-qubit rotation gates. This is sufficient for universal quantum computation. Later in the paper, we will be discussing qudit-based quantum computing and cluster state quantum computing using $S W A P^{\alpha}$ gates. In this section, we present our findings relating to a model of quantum computing that uses encoded quantum states as resource and that belong to the invariant subspaces of the symmetric group.

Divincenzo et al. [26] defined an encoded quantum computation model based on encoding three physical qubits in one logical qubit. For the case of qubits operated upon by the exchange interaction, we can have a different model of encoding and quantum computation that exploits the (permutation) symmetry of the system. For instance, for the three-qubit case, we can consider the 
invariant subspace [26] and Hamming weight 1 ,

$$
\alpha|1\rangle+\beta|2\rangle \stackrel{U}{\longrightarrow} \alpha^{\prime}|1\rangle+\beta^{\prime}|2\rangle
$$

where $U$ is an operation based on the symmetry of the invariant subspace, and $\alpha, \beta, \alpha^{\prime}$ and $\beta^{\prime}$ are complex numbers with $\sqrt{|\alpha|^{2}+|\beta|^{2}}=1$ and $\sqrt{\left|\alpha^{\prime}\right|^{2}+|\beta|^{2}}=1$. This is true for states with any number of qubits. If one were to start with a state that is a superposition of vectors within an invariant subspace and operate on it with an operator that abides by that symmetry, then the resultant output state will remain in the invariant subspace. The selection of invariant subspace depends on the number of vector states we want as our basis. This directly relates to the dimensionality of the invariant subspace.

Let us look at the kinds of initializations, operation and measurements that are required for this model of quantum computing.

\subsubsection{Initialization}

Ideally an input quantum state for a quantum computer is separable. However, in this model of invariant subspace-based, the input state must respect the symmetry of the invariant subspace. As a result, the input state can be the vector state, of the invariant subspace selected, which is closest to a separable state. For instance, for three-qubit states with the invariant subspace [26] and Hamming weight 1 , a good input state would be $|\psi\rangle=\frac{1}{\sqrt{2}}(|001\rangle-|100\rangle)$.

\subsubsection{Operations}

The operations that can be applied on the vector-state are selected based on the permutation symmetry of the invariant subspace. Firstly, we need to look at all the Youngs Tableaux [61] for the invariant subspace. Thereafter, we can decompose the cycle-structure into transpositions and apply associated $S W A P^{\alpha}$ on the vector state. The selection of the $S W A P^{\alpha}$ depends on the output state that is required.

\subsubsection{Measurements}

The measurement basis for the invariant-subspace based quantum computing model comprises the $m$ vector-states in the invariant subspace

$$
V=\left\{V_{1}, V_{2}, \cdots, V_{m}\right\}
$$

Theoretically, this measurement can be carried out by projection operators onto the vector states.

\subsection{Cluster State Quantum Computing}

Cluster states can be generated using $S W A P^{\alpha}$ gates [47] [62]. Tanamoto et al. [47] showed that this could be done for $\sqrt{S W A P}$ and iSWAP gates. We get to similar results independently, using numerical and analytical methods, and go on to define a dynamical model of cluster state quantum computation. Cluster states are pure quantum states [63] defined on two-level system arranged on a 
cluster-lattice. This cluster is a connected subset of a simple cubic lattice $\mathbb{Z}_{d}$ in $d \geq 1$ dimensions. The cluster states $\left|\phi_{\text {cluster }}\right\rangle$ obey the set of eigenvalue equations $M(a)\left|\phi_{\text {cluster }}\right\rangle=(-1)^{m_{a}}\left|\phi_{\text {cluster }}\right\rangle$ with the correlation operators $M(a)=\sigma_{x}^{(a)} \otimes \sigma_{z}^{(b)}$. Here, $b \in \operatorname{nbgh}(a)$, the set of all neighboring lattice sites of $a$, and $\left\{\right.$ cluster $\left._{m}\right\}:=\left\{m_{a} \in\{0,1\} \mid a \in\right.$ Cluster $\}$ is a set of binary parameters which specify the cluster state.

One of the fundamental differences that a $S W A P^{\alpha}$ model has with the usual CPHASE-based Raussendorf model of cluster quantum computation is that neighboring interactions generally do not commute: $\left[H_{i, i 1}, H_{i, i+1}\right] \neq 0$, unlike the CPHASE gate. As a result, we have, for the evolution operator,

$\mathrm{e}^{i H t} \neq \prod_{i, j} \mathrm{e}^{i H_{i, j} t}$. For creating cluster states using such non-commutative interactions, pairwise bonding between the qubits is needed. So, for an $n$-dimensional qubit array, cluster states can only be generated in $2 n$ steps: firstly, two-qubit cluster states are constructed by performing exchange interactions between pairs of nearest neighbor qubits. These qubit pairs are thereafter connected to each other using another set of such operations, and a onedimensional chain (cluster state) is created. Afterwards, these chains can be connected in various ways to give more complex structures, such as twodimensional clusters and ladder clusters. A point to remember here is that to reach the standard cluster-state form, as formulated by Raussendorf, single qubit rotation gates are required. Even though it is possible to have a modified $U_{\phi} U_{\psi}$ -based cluster state model, where $U_{\phi}$ and $U_{\psi}$ are measurement gates along an arbitrary angle, it is more convenient to change all bases to a standard form of two-qubit cluster states:

$$
|\psi\rangle_{C}^{2}=\left(|0\rangle_{1}|\rangle_{2}+|1\rangle_{1}|+\rangle_{2}\right)
$$

where $| \pm\rangle=\frac{1}{\sqrt{2}}(|0\rangle \pm|1\rangle)$. This realization can be carried out using a simple sequence of steps:

\subsubsection{Generating the Two-Qubit Cluster States}

Let us start with a state

$$
\left|\psi_{\text {in }}\right\rangle=|+\rangle_{1}|-\rangle_{2}
$$

Then, we apply a general $S W A P^{\alpha}$ gate:

$$
U_{S W A P^{\alpha}}\left|\psi_{i n}\right\rangle \rightarrow|0\rangle\left(|0\rangle \mathrm{e}^{i \pi \alpha}|1\rangle\right)+\mathrm{e}^{i \pi-i \pi \alpha}\left(|0\rangle+\mathrm{e}^{i \pi \alpha}|1\rangle\right)
$$

We use the following composite operator:

$$
U=\left(\begin{array}{cccc}
1 & 0 & 0 & 0 \\
0 & \mathrm{e}^{i \pi \alpha} & 0 & 0 \\
0 & 0 & \mathrm{e}^{i \pi \alpha} & 0 \\
0 & 0 & 0 & 1
\end{array}\right)
$$

to obtain the state $|\psi\rangle_{C}^{2}$. 


\subsubsection{Creating Larger Cluster States}

Let us start with the simple case of connecting two qubit-pairs with the states of the cluster states $C_{12}$ and a third qubit $|+\rangle_{3}$.

We can then apply the $S W A P^{\alpha}$ gate between qubits 2 and 3. This gives the state:

$$
\left|\psi_{0}\right\rangle=\left(|000\rangle+\mathrm{e}^{i \pi \alpha}|001\rangle-\mathrm{e}^{i \pi \alpha}|010\rangle-|011\rangle+|100\rangle+|101\rangle+|110\rangle+|111\rangle\right)
$$

We then operate with the operator:

$$
U=\left(\begin{array}{cccccccc}
1 & 0 & 0 & 0 & 0 & 0 & 0 & 0 \\
0 & \mathrm{e}^{i \pi \alpha} & 0 & 0 & 0 & 0 & 0 & 0 \\
0 & 0 & \mathrm{e}^{i \pi \alpha} & 0 & 0 & 0 & 0 & 0 \\
0 & 0 & 0 & 1 & 0 & 0 & 0 & 0 \\
0 & 0 & 0 & 0 & 1 & 0 & 0 & 0 \\
0 & 0 & 0 & 0 & 0 & 1 & 0 & 0 \\
0 & 0 & 0 & 0 & 0 & 0 & 1 & 0 \\
0 & 0 & 0 & 0 & 0 & 0 & 0 & 1
\end{array}\right)
$$

to obtain the cluster state:

$$
|\psi\rangle_{C}^{3}=(|+\rangle|0\rangle|+\rangle+|\rangle|1\rangle|\rangle)
$$

This method can be extended for higher number of qubits. As can be seen, a general $S W A P^{\alpha}$ has the problem of the need for the use of a non-local operator at the end to clean up the state to get the cluster state finally. This is, however, not the case for iSWAP gate, as used by Tanamoto et al. [47].

\subsection{Functional Quantum Computing}

In classical computing and programming, we have object-oriented and functional models of information processing. While the former deals with the manipulation of elements and resources using operators, the latter relates to the changing of operators to realize a certain computation. Classical functional computing relies on what is known as-calculus [64], which treats functions and data as the same type of objects. It allows for the computation of both functions of data as well as functions of functions.

This idea can be extended to the realm of quantum information processing too. The idea of quantum combs has been used for this purpose [65] [66], as have models based on quantum $\lambda$ calculus [67] [68]. The switch-based model of functional quantum computing [69] is the realization that we are most interested in. In this paper, we extended the idea from being a control-qubitbased model to a control-qudit-based model.

\section{The Model}

The model takes as its inputs a set of $N$ quantum operators $\left\{U_{0}, U_{1}, \cdots, U_{N-1}\right\}$, a control qudit $|d\rangle_{C}$ and a register of input qubits. In our model, let us consider that each operator is applied just once and that we encode the operator in the $N$-qubit control qudit as follows: $|0\rangle_{d}=|000 \cdots 0\rangle_{d}=|1234 \cdots(N-1)(N)\rangle_{e}$, 
$|1\rangle_{d}=|000 \cdots 1\rangle_{d}=|1234 \cdots(N)(N-1)\rangle_{e}$, $|2\rangle_{d}=|000 \cdots 10\rangle_{d}=|1234 \cdots(N-1)(N-2)(N)\rangle_{e}, \cdots$, $|N !\rangle_{d}=|111 \cdots 1\rangle_{d}=|(N)(N-1) \cdots 3421\rangle_{e}$. The encoding, marked by |\rangle$_{e}$ shows the sequence of the operators as well. The model coherently orders the quantum operators based on the value of the control-qudit, thereby creating a metaoperator, a superposition of many different sequential orderings of the same set of operators, which is then applied to the input qubit.

Let us call this entire operation $O_{S W A P}$ and let us take an illustration of this using the simple example of two operators $U_{1}$ and $U_{2}$. We will then have the control qudit in the states $|0\rangle_{d}=|12\rangle_{e}$ and $|1\rangle_{d}=|21\rangle_{e}$, which have the following action on the operators: $|0\rangle_{d} U_{1} U_{2}=U_{1} U_{2},|1\rangle_{d} U_{1} U_{2}=U_{2} U_{1}$. For a control qudit $|C\rangle=\alpha|0\rangle_{d}+\beta|1\rangle_{d}$ and an input state $|\psi\rangle$,

$$
\begin{aligned}
O_{S W A P}|c\rangle\left(U_{1} U_{2}\right)|\psi\rangle & =\alpha|0\rangle_{d}+\beta|1\rangle_{d}\left(U_{1} U_{2}\right)|\psi\rangle \\
& =\alpha|0\rangle_{d} U_{1} U_{2}|\psi\rangle+\beta|1\rangle_{d} U_{2} U_{1}|\psi\rangle
\end{aligned}
$$

This is an extremely useful tool for a multi-operator lattice-configuration such as the one we have developed for our model of cluster state quantum computation. Instead of restructuring the gates manually or even using gatepotentials in a synchronized manner, we can simply use the appropriate controlqudit to do the same.

\subsection{Qudit-Based Quantum Computing}

Quantum gates that are univesal for binary quantum logic operations belong to a family of unitary transforms are seen to be described by three parameters, and this arises out of the idea that up to an overall phase factor, any two dimensional unitary matrix can be written as

$$
U_{2}(\lambda, v, \phi)=\left(\begin{array}{cc}
\cos \lambda & -\mathrm{e}^{i v} \sin \lambda \\
\mathrm{e}^{i(\phi-v)} \sin \lambda & \mathrm{e}^{i \phi} \cos \lambda
\end{array}\right)
$$

expressed in the basis states $|0\rangle$ and $|1\rangle$. The three parameters are usually taken to be irrational multiples of $\pi$ and each other. This this allows even a single gate in to generate all single qubit transforms by repeated application. However, we find it to be more useful to consider these three parameters as arbitrary variables, with $U_{2}$ representing a family of gates that can be realized by an appropriate choice of three physical controls. One of the properties of $U_{2}$ is that it can transform any known state of a qubit to $|1\rangle: \alpha|0\rangle+\beta|1\rangle \stackrel{Z_{2}}{\longrightarrow}|1\rangle$. $U_{2}$ also contains the phase gate $X_{2}$ that alters the phase of $|1\rangle$ without affecting $|0\rangle:|1\rangle \stackrel{X_{2}}{\longrightarrow} \mathrm{e}^{i \phi}|1\rangle,|0\rangle \stackrel{X_{2}}{\longrightarrow}|0\rangle$. Using these two transformation properties of $U_{2}$, the two-qubit gates that are universal for quantum logic take the form

$$
A_{2}\left[U_{2}\right]=\left(\begin{array}{cc}
I_{2} & 0 \\
0 & U_{2}
\end{array}\right)
$$

The family of gates $A_{2}\left[U_{2}\right]$ is universal for binary quantum logic. A unitary 
transform on any number of qubits can be simulated by repeated application of these gates on just two qubits at any one given time.

We can generalize this to the multivalued case. Let us define $Z_{d}$ as a family of $d$-dimensional transforms that maps a known single-qudit state to $|d 1\rangle$ :

$$
\alpha_{1}|0\rangle+\alpha_{2}|1\rangle+\cdots+\alpha_{d-1}|d-1\rangle \stackrel{z_{d}}{\longrightarrow}|d-1\rangle
$$

Similarly, we can define the $d$-dimensional phase gate $X_{d}$ as a function that does the following:

$$
\begin{aligned}
& |d-1\rangle \stackrel{x_{d}}{\longrightarrow} \mathrm{e}^{i \phi}|d-1\rangle \\
& |q\rangle \stackrel{x_{d}}{\longrightarrow}|q\rangle, q \neq d-1
\end{aligned}
$$

We can now define the multivalued analog of $A_{2}\left[U_{2}\right]$ as

$$
A_{2}\left[U_{d}\right]=\left(\begin{array}{cc}
I_{d^{2}-d} & 0 \\
0 & U_{d}
\end{array}\right)
$$

For our system, we devise a very simple way to do this. We will present the formalism using a state with Hamming weight 1 for $n$-qubits. This can be generalized to other states with different Hamming weights. We define the state $|d-1\rangle$ as the n-qubit $W$-state:

$$
|d-1\rangle=|N-1\rangle=\frac{1}{\sqrt{N}}(|00 \cdots 01\rangle+|00 \cdots 010\rangle+\cdots+|100 \cdots 0\rangle)
$$

Now we define the other states by introducing a relative phase of $\mathrm{e}^{i \pi}$ in front of each of the superposition states one-by-one, so as to obtain the set of equations from

$$
|N-2\rangle=\frac{1}{\sqrt{N}}(|00 \cdots 01\rangle+|00 \cdots 010\rangle-|00 \cdots 0100\rangle+\cdots+|100 \cdots 0\rangle)
$$

to

$$
|0\rangle=\frac{1}{\sqrt{N}}(|00 \cdots 01\rangle \cdots+|010 \cdots 00\rangle-|100 \cdots 0\rangle)
$$

In this formalism, we will use the property that

$\frac{1}{\sqrt{2}}(|01\rangle+|10\rangle) \stackrel{\text { SWAP }^{\alpha}}{\longrightarrow} \frac{1}{\sqrt{2}}(|01\rangle+|10\rangle)$,

$\frac{1}{\sqrt{2}}(|01\rangle-|10\rangle) \stackrel{S W A P^{\alpha}}{\longrightarrow} \frac{\mathrm{e}^{i \pi \alpha}}{\sqrt{2}}(|01\rangle-|10\rangle)$. A point to note here is that even though we could have constructed $N$ logical qubits, we only constructed $N-1$. This was because the definition of $X_{d}$, as we do it below, does not allow the consideration of the state:

$\frac{1}{\sqrt{N}}(|00 \cdots 01\rangle|00 \cdots 010\rangle+|00 \cdots 0100\rangle+\cdots+|100 \cdots 0\rangle)$ for the properties of $X_{d}$ to hold true.

We define the $Z_{d}$ operator as an inverse map, based on the linear combination of vectors considered and 


$$
X_{d}=I_{2} \otimes I_{2} \otimes \cdots \otimes I_{2} \otimes U_{S W A P^{\alpha}}
$$

This allows us to construct the gate $A_{2}\left[U_{d}\right]$ and therefore do universal qudit quantum computation using our system. The composite gate can be constructed by applying individual gates, as mentioned in the tensor product, on the individual qubits or pairs of qubits. The only drawback of this is the construction of the inverse map for $Z_{d}$.

\section{Entanglement Swapping, Repeaters and Quantum Communication}

In the realm of quantum information processing, entanglement swapping plays a major role in helping generate entanglement in remote particles. Let us say we start with the $n$-qubit state: $\left|\psi_{\text {in }}\right\rangle=|010101 \cdots 01\rangle$. Now if we have pairs of qubits locally made to undergo exchange interaction, we shall get a series of entangled pairs. If the operator is the $\sqrt{S W A P}$ and we have even number of qubits, we will have $\left|\psi_{\text {int }}\right\rangle=\left(\frac{1+i}{2}|01\rangle+\frac{1-i}{2}|10\rangle\right) \ldots\left(\frac{1+i}{2}|01\rangle+\frac{1-i}{2}|10\rangle\right)$. Thus, we have qubit pairs $12,34,56,78, \cdots,(n-1, n)$. We found that if we now use exchange interaction on the pairs $23,45,67,89, \cdots,(n-2, n-1)$, and then perform single qubit measurements on all qubits from qubits 2 to $n-1$, we invariably entangle qubits 1 and $n$.

Interestingly, due to the symmetric way in which the state decomposition over vector states takes place, the entanglement between qubits 1 and $n$ is always maximal! Thus, using such smaller units (pairs) of entangled qubits, we can generate maximal entanglement over more complex structures and longer distances. Using this, we have found a variant of the conventional quantum repeater protocol by using exchange interaction instead of projective measurements. Even though both Bell-measurements (as used in conventional repeater protocols) and realization of exchange interaction (as in our model) have associated errors, our protocol is particularly useful for systems that give rise to the exchange interaction, such as in spin-systems and quantum dots. The maximally entangled state formed in our protocol between qubits 1 and $n$ can be used for various quantum information processing tasks. This is particularly useful for quantum communication protocols.

\subsection{Quantum Communication Protocols}

Quantum communication is the process of transferring an arbitrary quantum state from one place to another. One of its most important applications is Quantum Key Distribution (QKD), which is very important in quantum cryptography. Traditionally photonic systems have been the most popular for the realization of quantum communication. For the simple exchange of quantum information between the elements of a quantum information processing system over small distances, spin dynamics can help in realizing quantum communication protocols. 


\subsubsection{Stationary Qubit Model}

One of the most popular communication models in classical communication is the bus-based model. In this model, the bus/register is the primary unit of information processing and information is mediated between buses using flying bits. In the world of quantum information processing, this has traditionally been done by carrier particles such as photons. We can observe short-range and yet effective mediation done by the exchange interaction in spin-systems. In our stationary-qubit model, we have an integrated computing-and-communication system. Each computation unit comprises of an array of spins being driven through channels, such as electrons driven by surface acoustic waves in semiconductor heterostructures, and made to interact at specific locations in the system.

This leads to rapid development of entanglement in this computing unit. Now, we have a bunch of particles in an intermediate unit that is kept away from the computing unit, until they are required for mediating in the communication protocol. When this is so required, one particle from the computing bus-unit interacts with the flying qubit, which subsequently interacts with other flying qubits and finally with another computing bus-unit (and its qubits). In this manner, information is transferred from one computing bus-unit to another. A simple model in this case would be one where there are a finite number of flying qubits, say one, for instance. Let us tag this qubit as $F_{1}$. Let there be a target qubit in a second bus-unit, tagged $B_{2}$. Let the flying qubit $F_{1}$ and bus-qubit $B_{2}$ be initialized to $|+\rangle=\frac{1}{\sqrt{2}}(|0\rangle+|1\rangle)$. If the state on the qubit from the first bus (let us call it $B_{1}$ ) that is interacting with the flying qubit is in the state $\left|\psi_{B_{1}}\right\rangle=\alpha|0\rangle+\beta|1\rangle$, and we operate two distinct gates: $\operatorname{SWAP}^{\alpha_{1}}$ between $|\psi\rangle$ and $F_{1}$, and $S W A P^{\alpha_{2}}$ between $F_{1}$ and $B_{2}$, and we have

$$
\begin{aligned}
\left|\psi_{F_{1} B_{1} F_{2}}\right\rangle= & \frac{1}{2}\left(|0\rangle|0\rangle\left(\alpha|0\rangle+\left(\frac{3 \alpha}{4}+\frac{\beta}{4}+\left(\frac{\alpha}{4}-\frac{\beta}{4}\right)\left(\mathrm{e}^{i \pi \alpha_{1}}+\mathrm{e}^{i \pi \alpha_{2}}-\mathrm{e}^{i \pi\left(\alpha_{1}+\alpha_{1}\right)}\right)\right)|1\rangle\right)\right. \\
& +|0\rangle|1\rangle\left(\left(\frac{3 \alpha+\beta}{4}+\frac{\alpha+\beta}{4}\left(\mathrm{e}^{i \pi \alpha_{2}}-\mathrm{e}^{i \pi \alpha_{1}}\right)+\frac{\alpha-\beta}{4} \mathrm{e}^{i \pi\left(\alpha_{2}+\alpha_{1}\right)}\right)|0\rangle\right. \\
& \left.+\left(\frac{\alpha+\beta}{2}+\frac{\alpha-\beta}{2} \mathrm{e}^{i \pi \alpha_{1}}\right)|1\rangle\right)+|1\rangle|0\rangle\left(\left(\frac{\alpha+\beta}{2}+\frac{-\alpha+\beta}{2} \mathrm{e}^{i \pi \alpha_{1}}\right)|0\rangle\right. \\
& \left.\left.+\left(\frac{\alpha+3 \beta}{4}+\frac{\alpha+\beta}{4}\left(\mathrm{e}^{i \pi \alpha_{2}}-\mathrm{e}^{i \pi \alpha_{1}}\right)+\frac{-\alpha+\beta}{4} \mathrm{e}^{i \pi\left(\alpha_{2}+\alpha_{1}\right)}\right)\right)|1\rangle\right) \\
& +|1\rangle|1\rangle\left(\beta|1\rangle+\left(\frac{\alpha}{4}+\frac{3 \beta}{4}+\left(-\frac{\alpha}{4}+\frac{\beta}{4}\right)\left(\mathrm{e}^{i \pi \alpha_{1}}+\mathrm{e}^{i \pi \alpha_{2}}-\mathrm{e}^{i \pi\left(\alpha_{1}+\alpha_{1}\right)}\right)\right)|0\rangle\right)
\end{aligned}
$$

Perfect reconstruction of the system takes place for $\mathrm{e}^{i \pi \alpha_{1}}=\mathrm{e}^{i \pi \alpha_{2}}=-1$, as expected. This is the case of the SWAP gate. For all other $S W A P^{\alpha}$ gates, one needs to operate unitary operations on the $\left|\psi_{F_{2}}\right\rangle$ qubit, based on the selection of powers-of-SWAPs.

This model, however, has some associated problems: firstly, the tuning of the 
interaction for the flying qubits has to be very precise and localized to the area around a qubit in a manner that does not affect or influence the other flying qubits. As can be seen from the form of the state, there needs to be a great degree of control for this communication protocol. Secondly, errors could also arise with greater number of such interactions.

\subsubsection{Communication Using a Chain of Stationary Spins}

A natural extension that could take place would be if the interactions between the qubits in a chain are non-changing and not controllable, and we cannot apply any control fields to the qubits. Such systems where a large collection of spin are permanently coupled can be found in bulk materials. These mutual interactions of spins makes them either tend towards being aligned or antialigned with respect to each other, resulting in phenomena such as antiferromagnetism. The term spin chain describes a large class of materials wherein the spins are arranged in a one-dimensional lattice and are permanently coupled to each other (with the interaction strength decreasing with distance usually). In the spin-chain model, we can extend the communication model from the single qubit as initially defined to a collection of flying qubits that transmit a certain amount of information. We start this protocol with the initialization of the spin chain, say with $\left|\psi_{\text {in }}\right\rangle=|000000 \cdots 0\rangle$. I choose the couplings between the spins ( $s_{1}$ and $s_{2}$ ) of two qubits in the exchange interaction: $H=\sum_{i, j} J_{i, j} s_{i} \cdot s_{j}$ in such a manner that initialization of the spin chain to such a state is easy. For instance, if in the exchange interaction, we take the coupling constant such that $J<0$, we get the case of the ferromagnets, where the ground state in a magnetic field has all the spins oriented in the direction of this external field. Much like in the case of the single qubit mentioned above, in this slightly more involved protocol, a user Alice places an arbitrary quantum state at one end of the spin chain.

Let us say that Alice is on the $N^{\text {th }}$ site and Bob is on an arbitrary site $b$ on the site. For instance, if Alices state is $\frac{1}{\sqrt{2}}(|0\rangle+|1\rangle)$, then the state of the spin chain is $\left|\psi_{\text {in }}\right\rangle=\frac{1}{\sqrt{2}}(|000 \cdots 00\rangle+|000 \cdots 01\rangle)$. The natural evolution of this spin-chain leads to the state propagating as well being dispersed along the chain. Let us define the states $\left|\epsilon_{1}\right\rangle=|1000 \cdots 0\rangle,\left|\epsilon_{2}\right\rangle=|0100 \cdots 0\rangle, \cdots,\left|\epsilon_{N}\right\rangle=|000 \cdots 01\rangle$. Due to the Hamming-weight preserving symmetry of the exchange interaction, as discussed previously, the state $\left|\psi_{\text {in }}\right\rangle$ can only evolve into a superposition of the various $|\psi\rangle$ as defined above and $|000 \cdots 00\rangle$. As a result, the state of the spin changes at various points in the chain and also at Bob's end. Bob now has to choose an appropriate time to obtain a state that is as close to Alices state as possible: $\left|\psi_{\text {bob }}\right\rangle=\frac{1}{\sqrt{2}}\left(|000 \cdots 00\rangle+\left|\epsilon_{b}\right\rangle\right)$. The state of the spin at the site $b$ will, in general, be a mixed state. The resultant output state can be obtained by the partial tracing off of all the spins at the other sites. We can then find the final 
output state by evolving the initial state $\left|\psi_{\text {in }}\right\rangle$ :

$$
\left|\psi_{\text {out }}\right\rangle=\langle\phi| \mathrm{e}^{-i H t}\left(\frac{1}{\sqrt{2}}\left(|000 \cdots 00\rangle+\left|\epsilon_{N}\right\rangle\right)\right)|\phi\rangle
$$

where $|\phi\rangle$ are all possible $\mathrm{N}$ qubit states. Practically, in this example, only states with Hamming weight one will remain. The mixed state density matrix is given by partial trace with respect to the $N^{\text {th }}$ qubit: $=T r_{12 \cdots(N 1)}\left|\psi_{\text {out }}\right\rangle\left\langle\psi_{\text {out }}\right|$. The transition amplitude depends on the factor $\left\langle b\left|\mathrm{e}^{-i H t}\right| N\right\rangle$.

\section{Quantum Memory}

Computation, without memory, is not as optimal and efficient, and quantum computation is no different in the case of most algorithms and information processing tasks. This is particularly required in the context of quantum communication, and a way to realize this, which follows from the previous discussion on quantum communication protocols using a medium that has constant coupling constant that is always operational, has been formulated by us, as part of this project. Let us take the simple case of a system that has three components: the qubit(s) to be stored ( $Q_{A}$ at location $\mathrm{A}$ ), the memory bus and the qubit(s) in which the information is to be stored ( $Q_{M}$ at location $\left.\mathrm{M}\right)$. The simplest example would be when the initial states of qubits $Q_{A}$ and $Q_{M}$ are initialized as: $\left|\psi_{A}\right\rangle=|1\rangle,\left|\psi_{M}\right\rangle=|0\rangle$ and the bus-qubits in the state $\left|\psi_{B(1) B(2) \cdots B(N)}\right\rangle=|000 \cdots 000\rangle$. An important point to note here is that though the couplings $S_{B(i) B(j)}, i \neq j$ are operational always, the $S W A P^{\alpha}$-based couplings $S_{A B(1)}$ and $S_{B(N) M}$ are operated only when required.

We begin our protocol by switching on the couplings $S_{A B(1)}$ and $S_{B(N) M}$. Due to the Hamming weight symmetry of the $S W A P^{\alpha}$, the evolution of the states based on the couplings leads to a superposition of states with the same Hamming weight. We can select the couplings and time such that we reach a state as close to the quantum state: $\left|\psi_{A B(1) B(2) \cdots B(N) M}\right\rangle=|000 \cdots 001\rangle$. The simplest case in this is when the quantum-bus is represented by a single qubit. If we begin with switching on the coupling between $Q_{A}$ and the memory-bus, keeping the coupling between the memory-bus and QB switched off. One can realize the SWAP gate by continuous operation of the coupling giving a state

$\left|\psi_{A B(1)}\right\rangle=|01\rangle$. Now we shut off the coupling $S_{A B(1)}$ and switch on the one between the memory and the qubit $Q_{B}$. The SWAP gate is realized and the state is transferred to $Q_{B}$, completing the protocol. More complicated circuits and systems can be implemented, including those with multiple storage qubits attached to the memory-bus.

\section{Decoherence-Free Subspaces}

A decoherence-free subspace is a subspace of Hilbert space of a system that remains invariant to non-unitary dynamics [70] [71] [72]. The system is kept decoupled from the environment and therefore its evolution is completely unitary. Decoherence-free subspaces can be characterized as a special class of 
quantum error correcting codes (QECC), as shall be highlighted later in this chapter. These subspaces isolate quantum information and thereby prevent destructive or noisy interactions with the system's environment. These subspaces are an important conceptual and physical tool in quantum information, and are found to be useful when coherent control of a quantum system is required. Loss of coherence of quantum systems is called decoherence and takes place due to the interaction of a quantum system with uncontrollable degrees of freedom of the environment of the system. Since quantum computers cannot be truly and entirely isolated from their environment and thereby information can be lost due to decoherence, the study of decoherence-free subspaces is of utmost important for the implementation of quantum computation in the real world.

As per the definition of Decoherence-free subspaces [71], if we consider the dynamics of a system $S$ coupled to a bath $B$ and let the system evolve unitarily under the combined system-bath Hamiltonian

$$
H=H_{S} \otimes I_{B}+I_{S} \otimes H_{B}+H_{I}
$$

where $H_{S}$ and $H_{B}$ are the system and bath Hamiltonians respectively. $I_{S}$ and $I_{B}$ are the identity operator on the system and bath respectively. The last term in the hamiltonian denotes the interaction Hamiltonian

$$
H_{I}=\sum_{\alpha=1} S_{\alpha} \otimes B_{\alpha}
$$

where $S_{\alpha}$ and $B_{\alpha}$ act solely on the system and bath respectively.

The evolution in a subspace $\tilde{\mathcal{H}}$ of the system Hilbert space $\mathcal{H}$ is unitary for all possible bath states iff

1) The following condition holds true

$$
S_{\alpha}|\psi\rangle=a_{\alpha}|\psi\rangle, a_{\alpha} \in \mathbb{C}
$$

for all states $|\psi\rangle$ that span $\tilde{\mathcal{H}}$ and for every operator $S_{\alpha}$ in $H_{I}$.

2) Interaction operators $S$ and $B$ are decoupled initially.

3) $H_{S}|\psi\rangle$ has no overlap with states in the subspace that is orthogonal to $\tilde{\mathcal{H}}$.

Then the subspace $\tilde{\mathcal{H}}$ is called a decoherence-free subspace of $\mathcal{H}$.

Let us consider the dynamics of $N$ interacting spins that are collectively coupled to an environment with each spin experiencing the same interaction with its environment. We can then write $S_{\alpha}=\sum_{i} \sigma_{\alpha}^{(i)}$ with $\sigma_{\alpha}^{(i)}$ denoting operation on the $i^{\text {th }}$ qubit. If we expand these operators, they look like the following for an $N$-qubit case:

$$
\begin{aligned}
& S_{x}=\sigma_{x} \otimes I_{2 \times 2} \otimes \cdots \otimes I_{2 \times 2}+I_{2 \times 2} \otimes \sigma_{x} \otimes \cdots \otimes I_{2 \times 2}+\cdots+I_{2 \times 2} \otimes I_{2 \times 2} \otimes \cdots \otimes \sigma_{x} \\
& S_{y}=\sigma_{y} \otimes I_{2 \times 2} \otimes \cdots \otimes I_{2 \times 2}+I_{2 \times 2} \otimes \sigma_{y} \otimes \cdots \otimes I_{2 \times 2}+\cdots+I_{2 \times 2} \otimes I_{2 \times 2} \otimes \cdots \otimes \sigma_{y} \\
& S_{z}=\sigma_{z} \otimes I_{2 \times 2} \otimes \cdots \otimes I_{2 \times 2}+I_{2 \times 2} \otimes \sigma_{z} \otimes \cdots \otimes I_{2 \times 2}+\cdots+I_{2 \times 2} \otimes I_{2 \times 2} \otimes \cdots \otimes \sigma_{z}
\end{aligned}
$$

For the condition $S_{\alpha}|\psi\rangle=a_{\alpha}|\psi\rangle, a_{\alpha} \in \mathbb{C}$ to hold true for each of these forms of $S$, we must have states that will give a global and not local phase across the superposition in the operators $S_{x}, S_{y}$ and $S_{z}$. This is only possible if 
$|\psi\rangle=|+\rangle \otimes|+\rangle \otimes \cdots \otimes|+\rangle$ or $|-\rangle \otimes|-\rangle \otimes \cdots \otimes|-\rangle$ for the operator of the form in Equation (31), $|\psi\rangle=|+\rangle_{y} \otimes|+\rangle_{y} \otimes \cdots \otimes|+\rangle_{y}$ or $|-\rangle_{y} \otimes|-\rangle_{y} \otimes \cdots \otimes|-\rangle_{y}$ for the operator of the form in Equation (32), and $|\psi\rangle=|0\rangle_{y} \otimes|0\rangle_{y} \otimes \cdots \otimes|0\rangle_{y}$ or $|1\rangle_{y} \otimes|1\rangle_{y} \otimes \cdots \otimes|1\rangle_{y}$ for the operator of the form in Equation (33), where $| \pm\rangle=\frac{1}{\sqrt{2}}(|0\rangle \pm|1\rangle)$ and $| \pm\rangle_{y}=\frac{1}{\sqrt{2}}(|0\rangle \pm i|1\rangle)$. These can never be simultaneously true. As a result,

$$
S_{\alpha}|\psi\rangle=0
$$

Exponentiating the operators in (30) and using (34), we have

$$
O(\vec{p})=\exp \left[\sum_{\alpha}\left(S_{\alpha}-a_{\alpha} I\right) p_{\alpha}\right]|\psi\rangle=\exp \left[\sum_{\alpha}\left(-a_{\alpha} I\right) p_{\alpha}\right]|\psi\rangle
$$

If we now consider the hamiltonian for the exchange interaction:

$E|a\rangle|b\rangle \rightarrow|b\rangle|a\rangle$, we see that

$$
O(\vec{p}) E=\exp \left[\sum_{\alpha}\left(-a_{\alpha} I\right) p_{\alpha}\right] E=E \times \exp \left[\sum_{\alpha}\left(-a_{\alpha} I\right) p_{\alpha}\right]=E \times O(\vec{p})
$$

Thus, given the result in Equation (31), the operator given by the exchange interaction preserves the decoherence-free subspace for a "collective decoherence" model. The smallest number of physical qubits that gives a fully encoded Decoherence-free Subspace qubit is found to be four [71]. Let us take this case, and consider the states with zero angular momentum:

$$
\begin{gathered}
|0\rangle_{L}=\frac{1}{2}(|01\rangle-|10\rangle) \otimes(|01\rangle-|10\rangle) \\
|1\rangle_{L}=\frac{1}{\sqrt{3}}\left(|00\rangle \otimes|11\rangle-\frac{1}{2}(|01\rangle+|10\rangle) \otimes(|01\rangle+|10\rangle)+|11\rangle \otimes|00\rangle\right)
\end{gathered}
$$

Let us now look at the effect of the operation of the various exchange interactions $E_{i j}$, where the $i^{\text {th }}$ and $f^{\text {th }}$ qubits are being exchanged.

$$
E_{12}|0\rangle_{L} \rightarrow-|0\rangle_{L}, E_{12}|1\rangle_{L} \rightarrow|1\rangle_{L}
$$

Due to the symmetry of the logical basis states, $E_{34}$ has the same effect. Looking at the operation, we can define an encoded $\bar{Z}$ operator:

$$
\bar{Z}=-E_{12}=-E_{34}
$$

For defining a similar $\bar{X}$ operator: $\bar{X}|0\rangle_{L} \rightarrow|1\rangle_{L}, \bar{X}|1\rangle_{L} \rightarrow|0\rangle_{L}$ is not as straightforward since no one exchange interaction seems to provide the solution. Therefore, before moving forward with trying to define this composite operator, let us look at some other cases for the exchange interaction:

$$
\begin{aligned}
& E_{13}|0\rangle_{L} \rightarrow \frac{1}{2}(|0101\rangle-|1100\rangle-|0011\rangle+|1010\rangle)=\frac{1}{2}|0\rangle_{L}-\frac{\sqrt{3}}{2}|1\rangle_{L}, \\
& E_{13}|1\rangle_{L} \rightarrow \frac{1}{\sqrt{3}}\left(|1001\rangle-\frac{1}{2}(|0101\rangle+|1100\rangle+|0011\rangle+|1010\rangle)+|0110\rangle\right) \\
&=-\frac{\sqrt{3}}{2}|0\rangle_{L}-\frac{1}{2}|1\rangle_{L}
\end{aligned}
$$


Again, due to the symmetry of the states, the case for $E_{24}$ gives the same results. Using operators $E_{12}$ and $E_{13}$ (or $E_{34}$ and $E_{24}$ ), we can define the $\bar{X}$ operator

$$
\bar{X}=-\frac{1}{\sqrt{3}} E_{12}-\frac{2}{\sqrt{3}} E_{13}=-\frac{1}{\sqrt{3}} E_{34}-\frac{2}{\sqrt{3}} E_{24}
$$

The ability to implement these primary logical operations is sufficient to implement any gate in $S U(2)$ on the encoded qubits, by using the Euler angle reconstruction (about any two orthogonal axes):

$$
\exp (-i \omega(\vec{n} \cdot \vec{\sigma} / 2))=\exp \left(-i \beta \sigma_{z} / 2\right) \exp \left(-i \theta \sigma_{y} / 2\right) \exp \left(-i \alpha \sigma_{z} / 2\right)
$$

the resulting rotation is given by the angle $\omega$ about the direction specified by the unit vector $n$, both of which are functions of $\alpha, \beta$ and $\theta$. Mapping $\left(\sigma_{x}, \sigma_{y}, \sigma_{z}\right) \rightarrow(\bar{X}, \bar{Y}, \bar{Z})$, we can construct any element of $\mathrm{SU}(2)$ in the encoded space by turning on and off the appropriate exchange interaction.

For two qubit gates, we have to construct slightly more complex combinations of gates. Let us start with the controlled-Phase shift gate (CPHASE). The idea is to introduce a phase for the last case and not for any of the others. With some clever usage of the exchange interactions, this can be done:

$$
\left(-E_{12}-E_{56}\right)\left(-E_{12}-E_{56}-2 I\right)
$$

This gives us a phase only for the case for $|11\rangle_{L}$. The CPHASE gate has been previously realized in a different manner by Bacon et al. [71] using the operators: $h_{1}=\left[E_{26}, E_{12}+E_{25}\right]+\left[E_{15}, E_{12}+E_{16}\right], h_{2}=\sum_{j=5}^{8}\left(E_{1 j}+E_{2 j}\right)$ and $c=\frac{1}{32}\left[h_{1},\left(h_{2}, h_{1}\right)\right]$. As can be seen, our operator is a lot simpler in construction.

The CNOT gate can be realized similarly using two logical qubits. We find the form of this operator in the encoded space to be

$$
\frac{1}{\sqrt{3}}\left(I-E_{12}\right)\left(-E_{56}-2 E_{57}\right)
$$

The CNOT gate has been realized previously with two logical qubits comprising of three physical qubits as well by DiVincenzo et al. [26].

Thus, one can obtain a fault-tolerant universal set of gates using just the exchange interaction.

\section{Discussion}

Since the $S W A P^{\alpha}$ gates generated by the exchange interaction are non-commutative in general (adjacent operators operating on a common qubit), we can obtain an entire zoo of quantum states (and entanglement patterns) using these operators. However, given the parity constraints due to the permutation symmetries involved, we can define invariant subspaces for these states. This gives us an efficient method for classifying the generated states.

This resource and classification has been used for defining various models of quantum computation, quantum communication, quantum memory and deo- 
cherence-free subspaces. The Hamming-weight preserving symmetry of the $S W A P^{\alpha}$ gate is found to be of fundamental importance in most of these protocols. Due to the ubiquity of the exchange interaction in various physical systems including in condensed matter systems, this set of applications can be extremely useful in realizing quantum information processing in such physical systems.

\section{Conclusion}

In this paper, I have proposed new ways of applying entanglement generated using the exchange interaction for various quantum information processing tasks. This includes five distinct models of quantum computation, ways of implementing quantum communication protocols, quantum memory and defining decoherence-free subspaces. Future experimental realizations of quantum information processing that involve the exchange interaction can be based on the comprehensive theoretical study of these applications given in the paper.

\section{Acknowledgements}

We would like to acknowledge the contribution of Trinity College, Cambridge and the Nehru Trust for Cambridge University (NTCU) who funded the project. I would like to acknowledge Professor Crispin Barnes for helping with this paper.

\section{Conflicts of Interest}

The author declares no conflicts of interest regarding the publication of this paper.

\section{References}

[1] Einstein, A., Podolsky, B. and Rosen, N. (1935) Can Quantum-Mechanical Description of Physical Reality Be Considered Complete? Physical Review, 47, 777. https://doi.org/10.1103/PhysRev.47.777

[2] Schrödinger, E. (1935) Die gegenwärtige Situation in der Quantenmechanik. Naturwis-senschaften, 23, 807. https://doi.org/10.1007/BF01491891

[3] Horodecki, R., Horodecki, P., Horodecki, M. and Horodecki, K. (2009) Quantum Entanglement. Reviews of Modern Physics, 81, 865. https://doi.org/10.1103/RevModPhys.81.865

[4] Amico, L., Fazio, R., Osterloh, A. and Vedral, V. (2008) Entanglement in Many-Body Systems. Reviews of Modern Physics, 80, 517. https://doi.org/10.1103/RevModPhys.80.517

[5] Jurcevic, P., Lanyon, B.P., Hauke, P., Hempel, C., Zoller, P., Blatt, R. and Roos, C.F. (2014) Quasiparticle Engineering and Entanglement Propagation in a Quantum Many-Body System. Nature, 511, 202. https://doi.org/10.1038/nature13461

[6] Islam, R., Ma, R., Preiss, P.M., Tai, M.E., Lukin, A., Rispoli, M. and Greiner, M. (2015) Measuring Entanglement Entropy in a Quantum Many-Body System. Nature, 528, 77. https://doi.org/10.1038/nature15750

[7] Majumdar, M.G. (2018) Harnessing the Exchange Interaction for Quantum Infor- 
mation Processing. Preprints.

[8] Choi, K.S., Deng, H., Laurat, J. and Kimble, H. (2008) Mapping Photonic Entanglement into and out of a Quantum Memory. Nature, 452, 67.

https://doi.org/10.1038/nature06670

[9] Shadbolt, P.J., Verde, M.R., Peruzzo, A., Politi, A., Laing, A., Lobino, M., Matthews, J.C., Thompson, M.G. and O'Brien, J.L. (2012) Generating, Manipulating and Measuring Entanglement and Mixture with a Reconfigurable Photonic Circuit. Nature Photonics, 6, 45. https://doi.org/10.1038/nphoton.2011.283

[10] Weihs, G. (2001) Photonic Entanglement for Fundamental Tests and Quantum Communication. arXiv Preprint quant-ph/0107156.

[11] O’brien, J.L., Furusawa, A. and Vuckovic, J. (2009) Photonic Quantum Technologies. Nature Photonics, 3, 687. https://doi.org/10.1038/nphoton.2009.229

[12] Crocker, C., Lichtman, M., Sosnova, K., Nguyen, T., Carter, A., Inlek, V., Ruth, H. and Monroe, C. (2017) Photonic and Phononic Entanglement with Hybrid Species Ion Chains. 48 th Annual Meeting of the APS Division of Atomic, Molecular and Optical Physics, Sacramento, 5-9 June 2017.

[13] Chakram, S., Patil, Y. and Vengalattore, M. (2015) Multimode Phononic Correlations in a Nondegenerate Parametric Amplifier. New Journal of Physics, 17, Article ID: 063018. https://doi.org/10.1088/1367-2630/17/6/063018

[14] Busch, X., Carusotto, I. and Parentani, R. (2014) Spectrum and Entanglement of Phonons in Quantum Fluids of Light. Physical Review A, 89, Article ID: 043819. https://doi.org/10.1103/PhysRevA.89.043819

[15] Lukin, M., Yelin, S. and Fleischhauer, M. (2000) Entanglement of Atomic Ensembles by Trapping Correlated Photon States. Physical Review Letters, 84, 4232. https://doi.org/10.1103/PhysRevLett.84.4232

[16] Laurat, J., Choi, K., Deng, H., Chou, C. and Kimble, H. (2007) Heralded Entanglement between Atomic Ensembles: Preparation, Decoherence, and Scaling. Physical Review Letters, 99, 180504. https://doi.org/10.1103/PhysRevLett.99.180504

[17] Matsukevich, D., Chaneliere, T., Jenkins, S., Lan, S.-Y., Kennedy, T. and Kuzmich, A. (2006) Entanglement of Remote Atomic Qubits. Physical Review Letters, 96, Article ID: 030405. https://doi.org/10.1103/PhysRevLett.96.030405

[18] Hayes, D., Matsukevich, D., Maunz, P., Hucul, D., Quraishi, Q., Olmschenk, S., Campbell, W., Mizrahi, J., Senko, C. and Monroe, C. (2010) Entanglement Of atomic Qubits using an Optical Frequency Comb. Physical Review Letters, 104, 140501. https://doi.org/10.1103/PhysRevLett.104.140501

[19] Clark, S. and Parkins, A. (2003) Entanglement and Entropy Engineering of Atomic Two-Qubit States. Physical Review Letters, 90, Article ID: 047905. https://doi.org/10.1103/PhysRevLett.90.047905

[20] Kais, S. (2007) Entanglement, Electron Correlation, and Density Matrices. Advances in Chemical Physics, 134, 493. https://doi.org/10.1002/9780470106600.ch18

[21] Neder, I., Heiblum, M., Mahalu, D. and Umansky, V. (2007) Entanglement, Dephasing, and Phase Recovery via Cross-Correlation Measurements of Electrons. Physical Review Letters, 98, Article ID: 036803. https://doi.org/10.1103/PhysRevLett.98.036803

[22] Oh, S. and Kim, J. (2004) Entanglement of Electron Spins of Noninteracting Electron Gases. Physical Review A, 69, Article ID: 054305. https://doi.org/10.1103/PhysRevA.69.054305

[23] Jozsa, R. and Linden, N. (2003) On the Role of Entanglement in Quantum-Com- 
putational Speed-Up. Proceedings of the Royal Society of London A: Mathematical, Physical and Engineering Sciences, 459, 2011.

https://doi.org/10.1098/rspa.2002.1097

[24] Chitambar, E. and Hsieh, M.-H. (2016) Relating the Resource Theories of Entanglement and Quantum Coherence. Physical Review Letters, 117, Article ID: 020402. https://doi.org/10.1103/PhysRevLett.117.020402

[25] Bennett, C.H. and Di Vincenzo, D.P. (2000) Quantum Information and Computation. Nature, 404, 247. https://doi.org/10.1038/35005001

[26] Di Vincenzo, D.P. (2000) The Physical Implementation of Quantum Computation. Fort-schritte der Physik: Progress of Physics, 48, 771. https://doi.org/10.1002/1521-3978(200009)48:9/11<771::AID-PROP771>3.0.CO;2-E

[27] Ursin, R., Tiefenbacher, F., Schmitt-Manderbach, T., Weier, H., Scheidl, T., Lindenthal, M., Blauensteiner, B., Jennewein, T., Perdigues, J., Trojek, P., et al. (2007) Entanglement-Based Quantum Communication over $144 \mathrm{~km}$. Nature Physics, 3, nphys629. https://doi.org/10.1038/nphys629

[28] Majumdar, M.G. (2018) Quantum Correlations and Permutation Symmetries. Preprints.

[29] Penrose, R. (1998) Quantum Computation, Entanglement and State Reduction. Philosophical Transactions-Royal Society of London Series a Mathematical Physical and Engineering Sciences, 356, 1927-1939. https://doi.org/10.1098/rsta.1998.0256

[30] Sorensen, A. and Molmer, K. (2000) Entanglement and Quantum Computation with Ions in Thermal Motion. Physical Review A, 62, Article ID: 022311.

https://doi.org/10.1103/PhysRevA.62.022311

[31] Preskill, J. (2012) Quantum Computing and the Entanglement Frontier. arXiv Preprint ar-Xiv:1203.5813.

[32] Pan, J.W., Simon, C., Brukner, C. and Zeilinger, A. (2001) Entanglement Purification for Quantum Communication. Nature, 410, 1067.

https://doi.org/10.1038/35074041

[33] Aspelmeyer, M., Jennewein, T., Pfennigbauer, M., Leeb, W.R. and Zeilinger, A. (2003) Long-Distance Quantum Communication with Entangled Photons using Satellites. IEEE Journal of Selected Topics in Quantum Electronics, 9, 1541. https://doi.org/10.1109/JSTQE.2003.820918

[34] Bostrom, K. and Felbinger, T. (2002) Deterministic Secure Direct Communication using Entanglement. Physical Review Letters, 89, 187902. https://doi.org/10.1103/PhysRevLett.89.187902

[35] Jennewein, T., Simon, C., Weihs, G., Weinfurter, H. and Zeilinger, A. (2000) Quantum Cryptography with Entangled Photons. Physical Review Letters, 84, 4729. https://doi.org/10.1103/PhysRevLett.84.4729

[36] Naik, D., Peterson, C., White, A., Berglund, A. and Kwiat, P.G. (2000) Entangled State Quantum Cryptography: Eavesdropping on the Ekert Protocol. Physical Review Letters, 84, 4733. https://doi.org/10.1103/PhysRevLett.84.4733

[37] Grosshans, F., Cerf, N.J., Wenger, J., Tualle-Brouri, R. and Grangier, P. (2003) Virtual Entanglement and Reconciliation Protocols for Quantum Cryptography with Continuous Variables. arXiv Preprint quant-ph/0306141.

[38] Loss, D. and Di Vincenzo. D.P. (1998) Quantum Computation with Quantum Dots. Physical Review A, 57, 120. https://doi.org/10.1103/PhysRevA.57.120

[39] Imamog, A., Awschalom, D.D., Burkard, G., Di Vincenzo, D.P., Loss, D., Sherwin, M., Small, A., et al. (1999) Quantum Information Processing using Quantum Dot 
Spins and Cavity QED. Physical Review Letters, 83, 4204. https://doi.org/10.1103/PhysRevLett.83.4204

[40] Awschalom, D.D., Loss, D. and Samarth, N. (2013) Semiconductor Spintronics and Quantum Computation. Springer Science \& Business Media, Berlin.

[41] Neumann, P., Mizuochi, N., Rempp, F., Hemmer, P., Watanabe, H., Yamasaki, S., Jacques, V., Gaebel, T., Jelezko, F. and Wrachtrup, J. (2008) Multipartite Entanglement among Single Spins in Diamond. Science, 320, 1326.

https://doi.org/10.1126/science.1157233

[42] Childress, L. and Hanson, R. (2013) Diamond NV Centers for Quantum Computing and Quantum Networks. MRS Bulletin, 38, 134. https://doi.org/10.1557/mrs.2013.20

[43] Barnes, C., Shilton, J. and Robinson, A. (2000) Quantum Computation Using Electrons Trapped by Surface Acoustic Waves. Physical Review B, 62, 8410. https://doi.org/10.1103/PhysRevB.62.8410

[44] Zhou, Y., Zhang, G.F., Yang, F.H. and Feng, S.L. (2007) SWAP Operation in the Two-Qubit Heisenberg XXZ Model: Effects of Anisotropy and Magnetic Field. Physical Review A, 75, Article ID: 062304.

https://doi.org/10.1103/PhysRevA.75.062304

[45] Burkard, G., Loss, D., Di Vincenzo, D.P. and Smolin, J.A. (1999) Physical Optimization of Quantum Error Correction Circuits. Physical Review B, 60, 11404. https://doi.org/10.1103/PhysRevB.60.11404

[46] Barenco, A., C.H. Bennett, R. Cleve, D., Di Vincenzo, P., Margolus, N., Shor, P., Sleator, T., Smolin, J.A. and Weinfurter, H. (1995) Elementary Gates for Quantum Computation. Physical Review A, 52, 3457. https://doi.org/10.1103/PhysRevA.52.3457

[47] Tanamoto, T., Liu, Y.-X., Hu, X. and Nori, F. (2009) Efficient Quantum Circuits for One-Way Quantum Computing. Physical Review Letters, 102, 100501. https://doi.org/10.1103/PhysRevLett.102.100501

[48] Raussendorf, R. and Briegel, H.J. (2001) A One-Way Quantum Computer. Physical Review Letters, 86, 5188. https://doi.org/10.1103/PhysRevLett.86.5188

[49] Deutsch, D.E., Barenco, A. and Ekert, A. (1995) Universality in Quantum Computation. Proceedings of the Royal Society of London A, 449, 669. https://doi.org/10.1098/rspa.1995.0065

[50] Di Vincenzo, D.P. (1995) Two-Bit Gates Are Universal for Quantum Computation. Physical Review A, 51, 1015. https://doi.org/10.1103/PhysRevA.51.1015

[51] Shi, Y. (2002) Both Toffoli and Controlled-NOT Need Little Help to Do Universal Quantum Computation. arXiv Preprint quant-ph/0205115.

[52] Brylinski, J.-L. and Brylinski, R. (2002) Universal Quantum Gates. In: Mathematics of Quantum Computation, Chapman and Hall/CRC, London, 117-134. https://doi.org/10.1201/9781420035377.pt2

[53] Hsieh, M., Kempe, J., Myrgren, S. and Whaley, K.B. (2003) An Explicit Universal Gate-Set for Exchange-Only Quantum Computation. Quantum Information Processing, 2, 289. https://doi.org/10.1023/B:QINP.0000020084.53422.8e

[54] Schmidt-Kaler, F., Haffner, H., Riebe, M., Gulde, S., Lancaster, G.P., Deuschle, T., Becher, C., Roos, C.F., Eschner, J. and Blatt, R. (2003) How to Realize a Universal Quantum Gate with Trapped Ions. Nature, 422, 408. https://doi.org/10.1038/nature01494

[55] Rajagopal, A. and Rendell, R. (2002) Separability and Correlations in Composite States Based on Entropy Methods. Physical Review A, 65, Article ID: 032328. 
https://doi.org/10.1103/PhysRevA.66.022104

[56] Zanardi, P. (1999) Symmetrizing Evolutions. Physics Letters A, 258, 77. https://doi.org/10.1016/S0375-9601(99)00365-5

[57] Eltschka, C. and Siewert, J. (2012) Entanglement of Three-Qubit Greenberger-Horne-Zeilinger-Symmetric States. Physical Review Letters, 108, Article ID: 020502. https://doi.org/10.1103/PhysRevLett.108.020502

[58] Barenco, A., Berthiaume, A., Deutsch, D., Ekert, A., Jozsa, R. and Macchiavello, C. (1997) Stabilisation of Quantum Computations by Symmetrisation. SIAM Journal on Computing, 26, 1541. https://doi.org/10.1137/S0097539796302452

[59] Wang, X. and Zanardi, P. (2002) Simulation of Many-Body Interactions by Conditional Geo-Metric Phases. Physics Letters A, 301, 1. https://doi.org/10.1016/S0375-9601(02)00885-X

[60] Nielsen, M.A. and Chuang, I.L. (2004) Quantum Computation and Quantum Information (Cambridge Series on Information and the Natural Sciences). Cambridge University Press, Cambridge.

[61] Robinson, G.D.B. (1938) On the Representations of the Symmetric Group. American Journal of Mathematics, 60, 745-760. https://doi.org/10.2307/2371609

[62] Majumdar, M.G. and Barnes, C.H. (2016) Generation of Quantum Cluster States using Surface Acoustic Waves. arXiv Preprint arXiv:1605.08274.

[63] Raussendorf, R., Browne, D.E. and Briegel, H.J. (2003) Measurement-Based Quantum Computation on Cluster States. Physical Review A, 68, Article ID: 022312. https://doi.org/10.1103/PhysRevA.68.022312

[64] Barendregt, H. (1993) Lambda Calculi with Types, Handbook of Logic in Computer Science (vol. 2): Background: Computational Structures.

[65] Chiribella, G., DAriano, G.M. and Perinotti, P. (2008) Quantum Circuit Architecture. Physical Review Letters, 101, Article ID: 060401. https://doi.org/10.1103/PhysRevLett.101.060401

[66] Chiribella, G., D’Ariano, G.M. and Perinotti, P. (2009) Theoretical Framework for Quantum Networks. Physical Review A, 80, Article ID: 022339. https://doi.org/10.1103/PhysRevA.80.022339

[67] Van Tonder, A. (2004) A Lambda Calculus for Quantum Computation. SIAM Journal on Computing, 33, 1109. https://doi.org/10.1137/S0097539703432165

[68] Selinger, P. and Valiron, B. (2006) A Lambda Calculus for Quantum Computation with Classical Control. Mathematical Structures in Computer Science, 16, 527. https://doi.org/10.1017/S0960129506005238

[69] Rambo, T.M., Altepeter, J.B., Kumar, P. and D’Ariano, G.M. (2016) Functional Quantum Computing: An Optical Approach. Physical Review A, 93, Article ID: 052321. https://doi.org/10.1103/PhysRevA.93.052321

[70] Lidar, D.A., Chuang, I.L. and Whaley, K.B. (1998) Decoherence Free Subspaces for Quantum Computation. Physical Review Letters, 81, 2594. https://doi.org/10.1103/PhysRevLett.81.2594

[71] Bacon, D., Kempe, J., Lidar, D.A. and Whaley, K. (2000) Universal Fault-Tolerant Quantum Computation on Decoherence-Free Subspaces. Physical Review Letters, 85, 1758. https://doi.org/10.1103/PhysRevLett.85.1758

[72] Lidar, D.A. and Whaley, K.B. (2003) Decoherence-Free Subspaces and Subsystems. In: Irreversible Quantum Dynamics, Springer, Berlin, 83-120. https://doi.org/10.1007/3-540-44874-8_5 